\title{
STATIN INDUCED DYSURIA- A CASE REPORT
}

\author{
Radhika Soanker, Arun Jyothi, Sita ram.
}

\begin{abstract}
Statins are a class of hypolipidemic drugs, that are primarily used for the treatment of dyslipidemia and the prevention of cardiovascular disease. ATP III guidelines,2002, recommends that LDL cholesterol be the primary target of therapy, and lipid lowering therapy may be initiated based on evaluation of short term and long term cardiovascular risk(1). We are report a case of dysuria follow statin group of drugs, which is not enlisted in the side effect of these drugs. In the present case after re-challenge with similar group of drug patient again developed the symptoms. Underlying hyperlipidemia was effectively controlled with Fenofibrates.

KEY WORDS: Coronary Angiogram, Gender differences.
\end{abstract}

\section{INTRODUCTION}

Statins are a class of hypo-lipidemic drugs that inhibit the enzyme HMG-CoA reductase which plays a central role in the production of cholesterol. This enzyme catalyzes the conversion of HMG-CoA to mevalonate, an early and rate-limiting step in cholesterol biosynthesis. Drugs included in this class are: atorvastatin, rosuvastatin, fluvastatin, lovastatin, pitavastatin, pravastatin and simvastatin. Statins are primarily used for the treatment of dyslipidemia and the prevention of cardiovascular disease [1]. ATP III recommends that LDL cholesterol be the primary target of therapy and lipid lowering therapy may be initiated based on evaluation of short term and long term cardiovascular risk.

Here, we present the case of a female patient who was started on lipid lowering therapy with atorvastatin 5 yrs back, after careful evaluation of cholesterol profile and cardiovascular risk. She was a known hypertensive on treatment since 5yrs, which was controlled. Subsequently, she developed symptoms of dysuria and approached urologists which when was unresolved with empiric antibiotic treatment prescribed by local physician.

Article received on 25 March2016, published on 30 April 2016.

Radhika Soanker ${ }^{1}$ Arun Jyothi ${ }^{2}$ Sita ram ${ }^{3}$.

${ }^{1}$ Assistant Professor, Departments of clinical pharmacology, NIMS, India

${ }^{2}$ Senior resident, Department of clinical pharmacology

${ }^{3}$ Senior resident,Department of cardiology

Corresponding author: Radhika Soanker

Email: dr_radhika_s@yahoo.in

\section{CASE REPORT:}

A 56-year-old female patient presented to outpatient department of cardiology, with chest pain for which was carefully evaluated. After evaluation of lipid profile and cardiovascular risk (minimal CAD on coronary angiogram), she was started on Tablet Atorvastatin $10 \mathrm{mg}$ once daily at night. She was a known hypertensive on treatment with Losartan and Hydrochlorthiazide since 5yrs, which was controlled. After 15 days of treatment, she developed symptoms of dysuria. For which she took opinion of urologists which when was unresolved with empiric antibiotic treatment prescribed by local physician. She was symptomatically managed for dysuria, which was temporarily relieved with citralka syrup but failed to get permanent relief. She was thoroughly evaluated for dysuria, ultra sound abdomen, urine for culture at multiple occasions, cystoscopy, KUB abdomen and blood sugars, in which no significant abnormality was found. At the end of four years, one of the urologist whom she consulted had suspected atorvastatin induced dysuria and advised to stop it for time being. Subsequently, symptoms of dysuria gradually decreased and completely subsided in a week. After 6 months of stopping atorvastatin, patient came back to our institute for routine checkup. On evaluation, lipid profile revealed elevated LDL \& VLDL levels. Then the patient was started on Rosuvastatin 5mg once daily, after which she again developed symptoms of dysuria within one week and dysuria was controlled once the drug was stopped. Then, patient was started on fenofibrate which she has tolerated well.

\section{DISCUSSION:}

Atorvastatin is a HMG CoA reductase inhibitor[2] which is used to treat dyslipidemia [3] and to lower the risk of stroke, heart attack, or other heart complications in people with type 2 diabetes[4,5] coronary heart disease, or other risk factors. Atorvastatin is used in adults and children who are at least 10 years old.

Recent studies have shown that in patients suffering from acute coronary syndrome, high-dose statin treatment play a plaque-stabilizing role. At high doses, 
statins have anti-inflammatory effects, incite reduction of the necrotic plaque core, and improve endothelial function, leading to plaque stabilization and, sometimes, plaque regression. However, there is an increased risk of statin-associated adverse effects with such high-dose statin treatment[6]. There is a similar thought process and risks associated with using high-dose statins to prevent recurrence of thrombotic stroke[7].

Common side effects associated with atorvastatin are arthalgia, diarrhea, dyspepsia, myalgia, nausea [8] and some of the major side effects are myopathys [9], type 2 DM[10,11], Persistent liver enzyme abnormalities[12]. In 2014 the FDA reported memory loss, forgetfulness and confusion with all statin products including atorvastatin. The symptoms were not serious, and they were rare and reversible on cessation of drug treatment[10] Some case reports have shown lower urinary tract symptoms as adverse effect in statin users[13]

In present case, patient had dysuria for 4 year throughout the period when she was using atorvastatin and dysuria improved when the drug was discontinued. She developed dysuria again when rosuvastatin was started and dysuria improved when the drug was discontinued, so it could probably be statin induced. The mechanism responsible for these reactions is unknown. However, it was not a life threatening adverse event, but these adverse events should be monitored closely.

Dysuria is not enlisted as a side effect of atorvastatin. There are only few case reports which address this as a possible side effect of atorvastatin. Humberto J.et.al[14] in their case report mentioned the occurrence of Atorvastatin-Induced Hemorrhagic Cystitis. Fujimoto M et.al13 in their study which was a data mining of the public version of the FDA adverse event reporting system, showed small but reliable signals for Lower urinary tract symptoms in statin users.

Dysuria in this case was probably managed as a symptom of uncomplicated cystitis, which is most common in females, which afforded no relief to patient. A very high index of suspicion is needed to identify such drug induced side effects. Once it was identified as atorvastatin, she got complete relief in a week. Treatment for this adverse event includes, discontinuation of drug with symptomatic therapy and institution of different class of hypolipidemic drug.
Atorvastatin induced dysuria is a rare, uncommon side effect. Such rare adverse events should be suspected in all patients who develop similar symptoms on statin maintenance therapy. Prompt diagnosis and discontinuation of drug along with supportive care are crucial for the prognosis.

\section{REFERENCES}

1. National Cholesterol Education Program Expert Panel on Detection, Evaluation, and Treatment of High Blood Cholesterol in Adults (Adult Treatment Panel III). Circulation.2002; 106: 3143

2. Atorvastatin Calcium. Drugs.com. Retrieved 3 April 2011(unpublished data, from web site)

3. McCrindle BW, Ose L, Marais AD (July 2003). "Efficacy and safety of atorvastatin in children and adolescents with familial hypercholesterolemia or severe hyperlipidemia: a multicenter, randomized, placebo-controlled trial". J. Pediatr. 143 (1): 74-80.

4. Colhoun HM, Betteridge DJ, Durrington PN, Hitman GA, Neil HA, Livingstone SJ, Thomason MJ, Mackness MI, Charlton-Menys V, Fuller JH. Primary prevention of cardiovascular disease with atorvastatin in type 2 diabetes in the Collaborative Atorvastatin Diabetes Study (CARDS): multicentre randomised placebocontrolled trial". Lancet, 2004,364(9435): 685-96.

5. Jump up ${ }^{\wedge}$ Neil HA, DeMicco DA, Luo D, Betteridge DJ, Colhoun HM, Durrington PN, Livingstone SJ, Fuller JH, Hitman GA. Analysis of efficacy and safety in patients aged 65-75 years at randomization: Collaborative Atorvastatin Diabetes Study (CARDS). Diabetes Care, 2013,29 (11): 2378-84.

6. Rosa GM, Carbone F, Parodi A, Massimelli EA, Brunelli C, Mach F, Vuilleumier N, Montecucco F. Update on the efficacy of statin treatment in acute coronary syndromes. Eur. J. Clin.Invest,2014,44(5):501-15.

\section{CONCLUSION:}


7. Furie KL. High-dose statins should only be used in atherosclerotic strokes. Stroke, 2012,43,(7): 1994-5.

8. Lipitor: Prescribing Information. Pfizer. June 2009 (not unpublished).

9. Ghirlanda G, Oradei A, Manto A, Lippa S, Uccioli L, Caputo S, Greco AV, Littarru GP Evidence of plasma CoQ10-lowering effect by HMG-CoA reductase inhibitors: a double-blind, placebo-controlled study. JClin Pharmacol, 1993, 33 (3): $226-9$

10. FDA Expands Advice on Statin Risks. FDA, 31 January 2014, retrieved24 November 2015.

11. Boyles, Salynn. Statins May Raise Diabetes Risk in Older Women: Study: Middle-Aged, Older Statin Users Had More Type 2 Diabetes 2012,WebMD Health News, retrieved 24 November 2015.

12. LIPITOR (atorvastatin calcium) Tablets for oral administration"(PDF).Full Prescribing Information. Pfizer Inc. Retrieved 18 April 2014

13. Int J Clin Pharmacol Ther. 2014 Apr;52(4):259-66. doi: 10.5414/CP202033.Statin-associated lower urinary tract symptoms: data mining of the public version of the FDA adverse event reporting system, FAERS.Fujimoto $\mathrm{M}$, Hosomi K, Takada M

14. Humberto J. Martinez-Suarez,Rou Wang,Gary J. Faerber. Atorvastatin-Induced Hemorrhagic Cystitis: A Case Report Department of Urology, University of Michigan Medical School, Ann Arbor, Michigan, DOI: http://dx.doi.org/10.1016/j.urology.2008.03.027 